\title{
Disintegration Properties and Drug Release Profiles of Sodium Alginate Films Modified with Additives
}

\author{
Yoshifumi Murata*, Hitomi Sakano, Chieko Maida and Kyoko Kofuji \\ Faculty of Pharmaceutical Science, Japan \\ *Corresponding author: Yoshifumi Murata, Japan

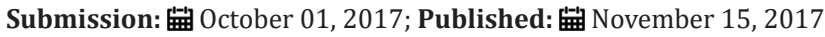

\begin{abstract}
The film dosage form (FD) is an attractive dosage form that can be used for local drug delivery. In this study, FDs were prepared with sodium alginate (Alg-Na) and additives. The disintegration profiles of the FDs were then assessed by measuring the amount of Alg-Na dissolved from the films in a limited aqueous medium. Caffeine and dexamethasone were used as the model drugs in the films. The relationship between disintegration rate of FD and drug release rate was then investigated. The disintegration rate of the FDs was accelerated when ascorbic acid was added as an additive to the film base. This phenomenon was not observed when sodium ascorbate was used as the additive. The disintegration profiles of the FDs did not affect the release rate of caffeine from the FDs. Conversely, the dissolution rate of dexamethasone increased when film disintegration was accelerated. The results show that Alg-Na-containing FDs are useful for patients due to their high solubility in limited media.
\end{abstract}

Keywords: Film dosage form; Sodium alginate; Film disintegration; Drug release profile

\section{Introduction}

Film dosage forms (FDs) are films that contain active compounds. They are an attractive dosage form that are prepared using water-soluble polymers that quickly swell and disintegrate in bodily fluids such as saliva. The incorporated active compound is released from the gel matrix as the FD disintegrates. Therefore, the FD is an excellent tool by which drugs can be delivered to local disease sites in the oral cavity [1,2]. FDs can simplify drug administration and may be useful for patients who have difficulty swallowing conventional oral dosage forms [3-5].

A water-soluble polysaccharide is a material that forms a thin film after the solvent in its solution is evaporated. Sodium alginate (Alg-Na) is a natural polysaccharide that is of interest because of its safety for oral administration. FDs can be simply prepared with high-molecular-weight Alg-Na using the casting method without the need for dissolution in organic solvents or heating. It has been reported that some Alg-Na-based FDs are useful for treating localized conditions in the oral cavity, such as stomatitis and oral mucositis [6,7].

The disintegration rate of a film matrix is an important factor when characterizing FDs; however, the degree of erosion is difficult to quantify $[8,9]$. We previously reported on a simple colorimetric assay to measure the amount of Alg- $\mathrm{Na}$ in aqueous solution [10]. This method was subsequently used to generate disintegration profiles in the test medium for Alg-Na-containing FDs. The disintegration profiles of the FDs differed depending on the Alg-Na type used as the film base.
In the present study, FDs were prepared using Alg-Na solutions containing additives as the film base. Sodium glucoronate, glucuronic acid, ascorbic acid, and sodium ascorbate were used as additives in the FDs. The disintegration profiles of the films were then assessed by measuring the amount of Alg-Na dissolved from each FD. Finally, the relationship between disintegration rate of FD and drug release in limited aqueous medium was investigated.

\section{Experimental}

\section{Materials}

Three types of Alg-Na were used as the film bases: Alg-A (300cps; Nacalai Tesque Inc., Kyoto, Japan), Alg-B (500cps, Nacalai Tesque Inc.), and Alg-C (150M; Kibun Food Chemifa Co., Tokyo, Japan). Hydroxylamine (HX), caffeine (CAF), and dexamethasone (DM) were purchased from Wako Pure Chemical Industries, Ltd. (Osaka, Japan). CAF and DM were used as model drugs. 1-Cyclohexyl-3-(2morpholinoethyl) carbodiimide metho-p-toluenesulfonate (CMEC) was purchased from Aldrich Chemical Co. (Milwaukee, WI, USA). All other chemicals were of reagent grade and were obtained from commercial sources.

\section{Preparation of alginic acid (ALG)}

ALG was prepared as follows. Briefly, $2 \mathrm{M} \mathrm{HCl}$ was gradually added to $30 \mathrm{~g}$ Alg-Na solution $(1 \% \mathrm{w} / \mathrm{w})$ while stirring and cooling down with ice water. A deposit caused by the coacervation occurred when the $\mathrm{pH}$ of the solution was reduced to below 3 . The coacervate was centrifuged at $1900 \times$ g ( 2100 model; Kubota Co., Tokyo, Japan) 
for $5 \mathrm{~min}$, followed by washing once with ion-exchanged water, washing three times with ethanol, and drying. The white block obtained was ground with a pestle to obtain alginic acid powder, which was sieved (mesh size, $75 \mu \mathrm{m}$ ) before use in subsequent experiments.

\section{FD preparation}

FD was prepared as follows: $1.5-3.0 \%(\mathrm{w} / \mathrm{w})$ Alg-Na containing an additive such as ALG was dispersed in deionized water to prepare the film base solution. The mixture was thoroughly mixed by sonication and poured ( $3 \mathrm{~g}$ each) into individual plastic petri dishes (diameter, $54 \mathrm{~mm}$ ). The dishes were kept for $24 \mathrm{~h}$ at $37{ }^{\circ} \mathrm{C}$, after which the circular films formed were transferred into a desiccator. Drug-containing FDs were also prepared with the AlgNa solution by the same method.

\section{Solubility of model drug}

The solubility of each model drug was measured in physiological saline. The drug and an additive were added to the test solution and shaken at $37{ }^{\circ} \mathrm{C}$ for $24 \mathrm{~h}$. The suspension was then removed using a pre-heated plastic syringe (Terumo Co., Tokyo, Japan) at $37{ }^{\circ} \mathrm{C}$ and filtered using a syringe-driven filter unit (Millex-HV; pore size, $0.45 \mu \mathrm{m}$; Millipore Co., Danvers, MA, USA). The filtrate was diluted with physiological saline and injected onto a high-performance liquid chromatography (HPLC) column for analysis.

\section{Assay of model drugs}

The HPLC system (Hitachi Co., Tokyo, Japan) consisted of a pump (L-2130), UV-detector (L-2400), autosampler (L-2200), and chromate-integrator (D-2500) connected to a packed column (150mm $\times 4.6 \mathrm{~mm}$, Cosmosil 5C18-MS-II, Nacalai Tesque Inc.). To determine the concentration of $\mathrm{CAF}$, the analysis was conducted at ambient temperature using a mobile phase consisting of $50 \mathrm{mM}$ phosphate buffer ( $\mathrm{pH}$ 4.0) and methanol (81:19) at a flow rate of $1.2 \mathrm{ml} / \mathrm{min}$ [11]. The detector wavelength was set at $215 \mathrm{~nm}$. For the analysis of DM, the mobile phase consisted of $10 \mathrm{mM}$ phosphate buffer ( $\mathrm{pH} 2.3$ ) and acetonitrile (13:7) at a flow rate of $1.0 \mathrm{ml} / \mathrm{min}$. The detector wavelength was set at 230nm [12].

\section{Film disintegration test}

A film was placed in a plastic dish and $10 \mathrm{ml}$ of physiological saline preheated to $37{ }^{\circ} \mathrm{C}$ was added. The dish was then shaken (300rpm) in an incubator (SI-300; As One Co., Osaka, Japan) set at $37^{\circ} \mathrm{C}$. The medium $(0.3 \mathrm{ml})$ was periodically removed using a plastic syringe and filtered through a syringe-driven filter unit (pore size, $0.45 \mu \mathrm{m})$. An equal volume $(0.3 \mathrm{ml})$ of physiological saline at 37 ${ }^{\circ} \mathrm{C}$ was added to the dish in the incubator to maintain a constant volume. Aliquots $(0.2 \mathrm{ml})$ of the filtered solution were combined with $0.8 \mathrm{ml}$ of ion-exchanged water in test tubes before being mixed well with the aid of a vortex mixer. The amount of Alg-Na in each sample solution ( $1 \mathrm{ml}$ ) was measured using the method described below. Each test was performed in triplicate.

\section{Colorimetric assay of alginate}

The reagent solutions used were $20 \mathrm{mM}$ HX in ion-exchanged water and 0.1 M CMEC in 2\% pyridine-HCl buffer ( $\mathrm{pH}$ 5.0). Aliquots (1ml) of HX and CMEC reagents were added to $1 \mathrm{ml}$ of the sample solution, followed by vortex mixing. Each mixture was incubated at $40{ }^{\circ} \mathrm{C}$ for $20 \mathrm{~min}$, after which $20 \mathrm{mM} \mathrm{FeCl} 3$ in $0.1 \mathrm{M} \mathrm{HCl}(3 \mathrm{ml})$ was added. Absorbance of the solution in a quartz cell ( $1 \mathrm{~cm}$ light path) was measured at 480nm using a spectrophotometer (UV-1200; Shimadzu, Kyoto, Japan). Each absorbance value was normalized to that of a blank reagent. For each test, a calibration curve was constructed using a fresh set of Alg-Na standards.

\section{Drug dissolution test}

The sample solution was obtained by the same method described in the film disintegration test section. Next, 80- $\mu$ l aliquots of the filtered sample solution were placed in micro test tubes $(1.5 \mathrm{ml})$, to which $720 \mu \mathrm{l}$ of methanol was added to precipitate the polysaccharide dissolved from the dosage form. Samples were mixed and centrifuged (7,700×g, 5min; H-1300; Kokusan Co., Saitama, Japan), and the supernatants were injected onto the HPLC column. Each test was performed in triplicate.

\section{Results and Discussion}

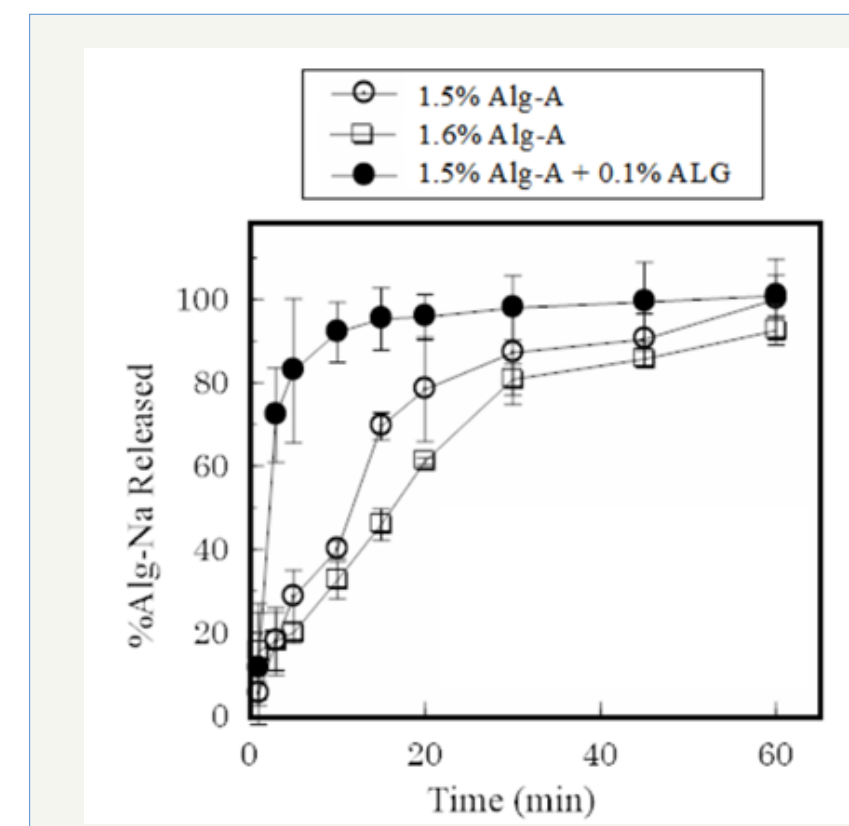

Figure 1: Dissolution profiles of Alg-Na from FDs prepared with Alg-A.

The FDs swelled and disintegrated, resulting in Alg-Na release into the aqueous medium (Figure 1). For the FD prepared with $1.6 \%$ Alg-A, $33 \pm 4 \%$ of the incorporated Alg-Na dissolved by $10 \mathrm{~min}$ and about $80 \%$ of the film base dissolved into the test medium at 30min. Films containing ALG showed an accelerated disintegration rate. For example, $92 \pm 7 \%$ of the incorporated Alg-Na dissolved after 10min from the FD prepared with 1.5\% Alg-A and 0.1\% ALG (obtained from Alg-A). This phenomenon was not observed for the FD prepared with $1.6 \%$ Alg-A.

As shown in Figure 2, disintegration rate increased when $0.1 \%$ ascorbic acid was added to the film base solution. Furthermore, 
Alg-Na quickly dissolved in the test solution, and the total amount of Alg-Na contained in the FD dissolved after $5 \mathrm{~min}$. Accelerated disintegration was not observed for the FD modified with $0.1 \%$ sodium ascorbate. Glucuronic acid changed the disintegration profiles of the FDs, whereas sodium glucuronate did not. These results show that the presence of not only an additive containing carboxylic acid, but also a weak acid such as ascorbic acid, can affect the disintegration of FDs.

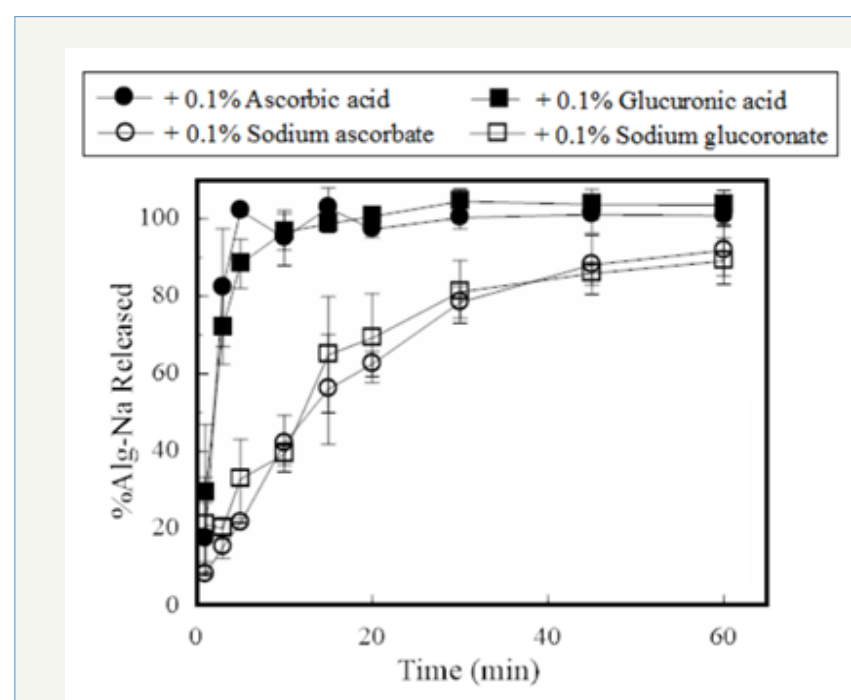

Figure 2: Dissolution profiles of Alg-Na from FDs modified with additives.

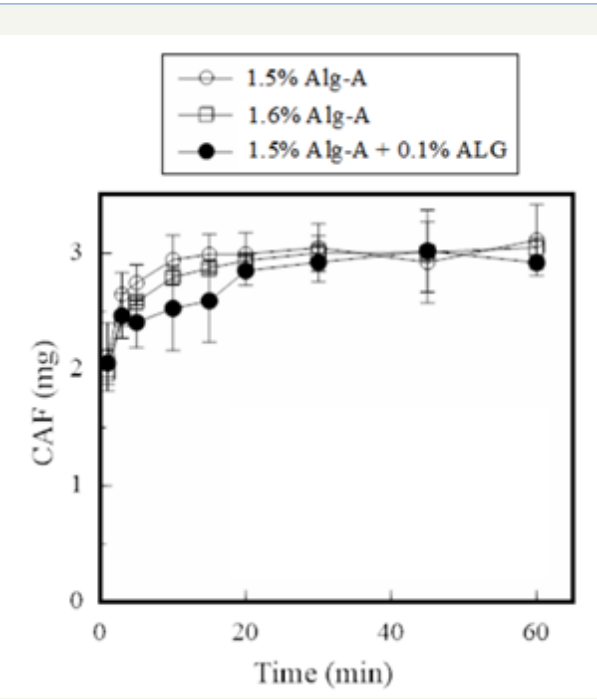

Figure 3: Release profiles of CAF from FDs prepared with Alg-A.

CAF is a water-soluble compound. It showed a solubility of $25 \mathrm{mg} / \mathrm{ml}$ in physiological saline at $37{ }^{\circ} \mathrm{C}$. CAF dissolved immediately the FD containing it was brought in contact with the test medium. As shown in Figure 3, more than $2 \mathrm{mg}$ of CAF dissolved at $1 \mathrm{~min}$ from the FD prepared with $1.6 \%$ Alg-A; however, almost all of the CAF incorporated in the FD was released after 10min. A rapid dissolution profile for CAF was also observed for the FD modified with $0.1 \%$ ALG (obtained from Alg-A). DM is slightly soluble in water. It was observed that DM gradually dissolved from the FD in the aqueous medium. As shown in Figure 4, $0.30 \pm 0.02 \mathrm{mg}$ of DM had dissolved by $10 \mathrm{~min}$, whereas $77 \%$ of the total DM incorporated in the FD dissolved after $45 \mathrm{~min}$. After addition of ALG to the film base, there was an apparent increase in the initial dissolution rate of DM from the FD. For example, $0.52 \pm 0.05 \mathrm{mg}$ of DM had dissolved by $10 \mathrm{~min}$ from the FD prepared with $1.5 \%$ Alg-A and $0.1 \%$ ALG, which corresponds to about $70 \%$ of the total DM incorporated in the FD. Increment in drug dissolution rate was also observed for FDs modified with other additives. It was noted that $0.57 \mathrm{mg}$ of DM had dissolved at 10min from FDs containing 1.5\% Alg-A and either $0.1 \%$ ascorbic acid or $0.1 \%$ glucuronic acid. However, this was not observed when the FD was prepared with the film base solution containing sodium ascorbate or sodium glucuronate.

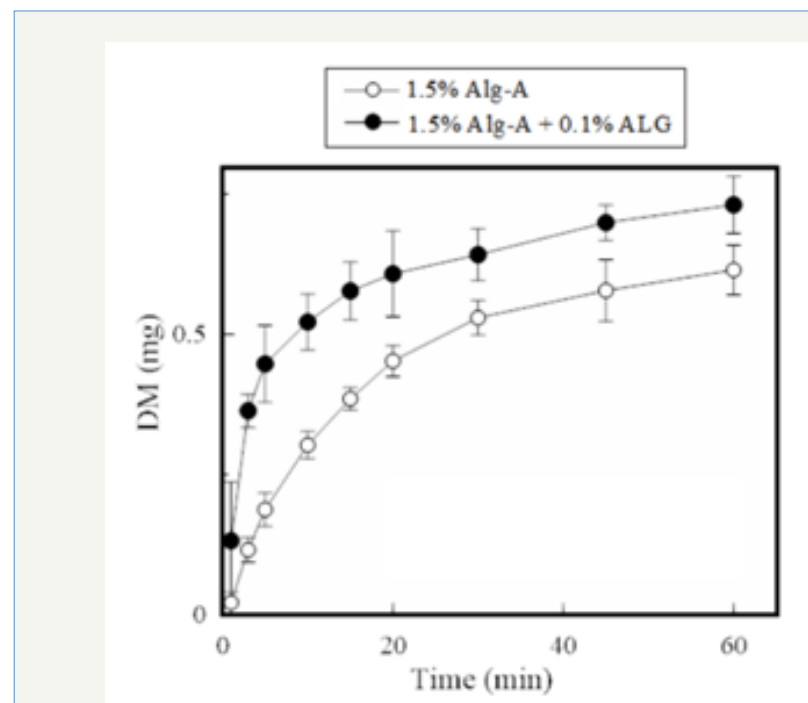

Figure 4: Release profiles of DM from FDs prepared with Alg-A.

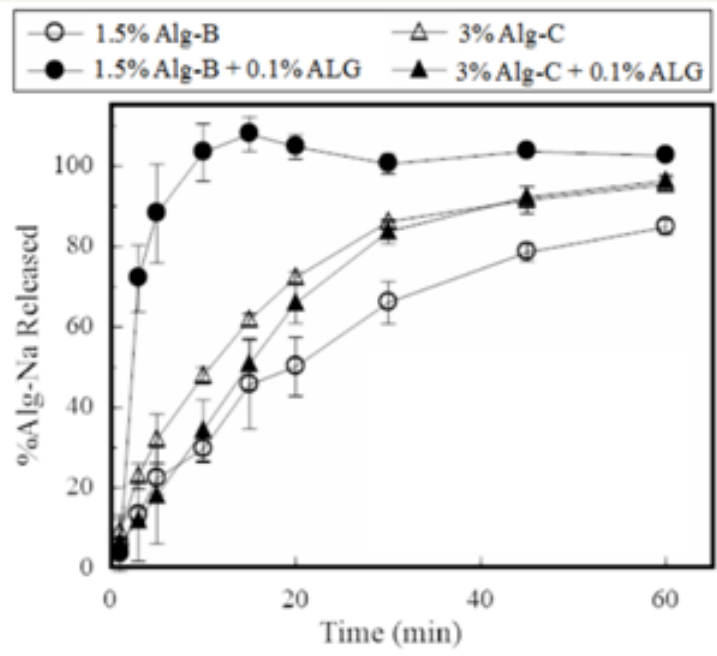

Figure 5: Dissolution profiles of Alg-Na from FDs prepared with Alg-B or Alg-C.

Figure 5 shows the film disintegration profile of FD prepared with 1.5\% Alg-B. Accelerated film disintegration was observed for 
the FD modified with $0.1 \%$ ALG. In addition, the dissolution rate of DM from the FD was increased by the modification, as shown in Figure 6. Conversely, neither accelerated film disintegration nor increased DM dissolution was noted for the FD prepared with 3\% Alg-C containing $0.1 \%$ ALG. It has been reported that modification of an FD with a surface-active agent accelerates drug dissolution from the film because the additive increases drug solubility in water [13]. DM solubility did not change after ALG or ascorbic acid was added to the formulation (Table 1). These results show that modification of FDs using additives affects the disintegration of the film matrix. This was evident from the change in the initial dissolution rate of DM from the dosage form.

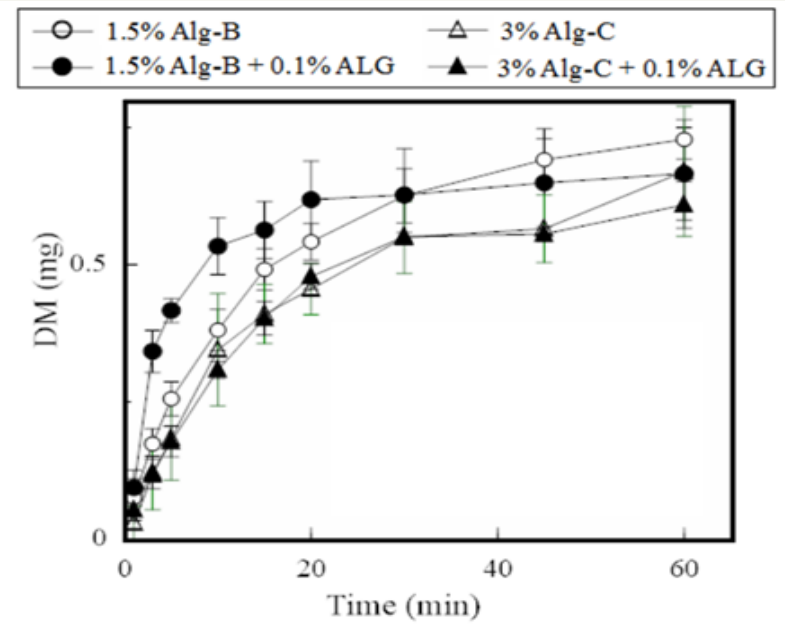

Figure 6: Release profiles of DM from FDs prepared with Alg-B or Alg-C.

Table 1: Solubility of DM in physiological saline at $37^{\circ} \mathrm{C}$.

\begin{tabular}{|c|c|}
\hline Additive & Solubility $(\boldsymbol{\mu g} / \mathbf{m l})$ \\
\hline None & 78 \\
\hline $0.03 \%$ Ascorbic acid & 80 \\
\hline $0.03 \%$ ALG $^{*}$ & 76 \\
\hline
\end{tabular}

\section{Conclusion}

FDs containing therapeutic agents are usually prepared as fastdissolving dosage forms for drug dissolution in limited biological media. In the present study, disintegration profiles and the rates of drug release from FDs prepared with Alg-Na solution containing additives as film bases were investigated. FD disintegration increased after the formulation was modified by the addition of additives. In addition, FD erosion affected the dissolution rate of the water-insoluble model drug DM from the dosage forms. Our findings show that FDs prepared with Alg-Na may be useful dosage forms for patients due to their high solubility even in restricted amounts of media, such as saliva. Furthermore, drug release rate is controllable by modification of the film base with suitable additives.

\section{References}

1. Kassem AA, Issa DA, Kotry GS, Farid RM (2017) Thiolated alginate-based multiple layer mucoadhesive films of metformin forintra-pocket local delivery: in vitro characterization and clinical assessment. Drug Dev Ind Pharm 43(1): 120-131.

2. Hanif M, Zaman M (2017) Thiolation of arabinoxylan and its application in the fabrication of controlled release mucoadhesive oral films. Daru 25(1): 6.

3. Okabe H, Suzuki E, Sugiura Y, Yanagimoto K, Takanashi Y, et al. (2008) Development of an easily swallowed film formulation. Int J Pharm 355(1-2): 62-66.

4. Satyanarayana DA, Keshavarao KP (2012) Fast disintegrating films containing anastrozole as a dosage form for dysphagia patients. Arch Pharm Res 35(12): 2171-2182.

5. Orlu M, Ranmal SR, Sheng Y, Tuleu C, Seddon P (2017) Acceptability of orodispersible films for delivery of medicines to infants and preschool children. Drug Deliv 24(1): 1243-1248.

6. Shi LL, Xu WJ, Cao QR, Yang M, Cui JH (2014) Preparation, characterization and in vitro evaluation of a polyvinyl alcohol/sodium alginate based orodispersible film containing sildenafil citrate. Pharmazie 69(5): 327334.

7. Murata Y, Kofuji K, Nishida N, Kamaguchi R (2012) Development of film dosage form containing allopurinol for prevention and treatment of oral mucositis. ISRN Pharm 2012(2012): 1-5.

8. Laitinen R, Räty J, Korhonen K, Ketolainen J, Peiponen KE (2017) Reflectometric monitoring of the dissolution process of thin polymeric films. Int J Pharm 523(1): 127-132.

9. Lindert S, Breitkreutz J (2017) Oromucosal multilayer films for tailormade, controlled drug delivery. Expert Opin Drug Deliv 14(11): 12651279.

10. Murata Y, Nakayama A, Sasaki S (2016) Disintegration properties of films prepared with sodium alginate: A colorimetric quantification method. J J Mater Sci 1(1): 1.

11. Acheampong A, Gyasi WO, Darko G, Apau J, Addai-Arhin S (2016) Validated RP-HPLC method for simultaneous determination and quantification of chlorpheniramine maleate, paracetamol and caffeine in tablet formulation. Springer plus 14(5): 625-632.

12. Kumar V, Mostafa S, Kayo MW, Goldberg EP, Derendorf H (2006) HPLC determination of dexamethasone in human plasma and its application to an in vitro release study from endovascular stents. Pharmazie 61(11): 908-911.

13. Murata Y, Isobe T, Kofuji K, Nishida N, Kamaguchi R (2013) Development of film dosage forms containing miconazole for the treatment of oral candidiasis. Pharmacology \& Pharmacy 4: 325-330. 